\title{
A STUDY OF DIPHTHERIA BACILLI WITH SPECIAL REFERENCE TO COMPLEMENT-FIXATION REACTIONS.*
}

\author{
JOHN A. KOLMER.
}

(From the Laboratory of Experimental Pathology, Universily of Pennsylvania, Philadelphia.)

Because of the important relation of diphtheria to public health and the ease with which cultures usually may be made from the infected parts, the bacteriological diagnosis of diphtheria soon became an important adjunct in the diagnosis and management of this infection. It was soon discovered, however, that the bacteriology of diphtheria was not a simple matter, but that the bacillus might present a most varied morphology. Quickly a "group" of organisms was isolated, which centered about a chief type but differed in certain details.

The study of this group and especially the identity of the Hofmann bacillus and its relation to the diphtheria bacillus has engaged the attention of bacteriologists ever since it was discovered in I886. Whether or not this pseudo-type could be made virulent or change its morphology to resemble the typical diphtheria bacillus, and vice versa, whether a true diphtheria bacillus could be made to resemble the pseudo-type, were problems which called forth extensive research with varied results. That the question is one of great importance is readily understood. This study bears specifically upon this question.

Among the first experiments carried out by Bordet and Gengou in their studies upon complement-fixation was the use of a bacterial antigen (plague) and its immune serum. It was found that complement was fixed in the presence of antigen and immune body but remained free if either of these were absent. A specific relation was supposed to exist between antigen and its immune body, and accordingly Wassermann, in evolving his practical sero-diagnosis of syphilis, used tissues rich in Treponema pallidum. Subsequently it was found that normal organs would suffice as antigens and

* Received for publication April 24, Igr2. 
that the lipoids are the active agents in such antigens, being brought into relationship with the immune body of the syphilitic serum through the agency of the globulins (Adami).

The reaction has been applied to the diagnosis of diseases other than syphilis by the substitution of specific antigens, notably in glanders and gonorrhea. The reaction of complement-fixation depends chiefly upon the fact that neither antigen nor immune body alone can fix complement, and that this fixation occurs only when both are present in certain proportions. Consequently if the antigen is known the immune body may be found, or conversely, if the immune body is known the antigen may be found.

With others we have been able to show that to a certain extent streptococci may be differentiated by complement-fixation reactions with homologous antigens. ${ }^{r}$ The question then occurred to us whether or not it is possible to differentiate between the various types of diphtheria bacilli and in particular between the true diphtheria bacillus and the Hofmann's bacillus, by complementfixation reactions with homologous antigens and immune sera. If, for instance, the serum of an animal immunized with a true diphtheria bacillus did not fix complement with an antigen of Hofmann's bacillus but did so with its own antigen, it would tend to show that the two organisms were different species. On the other hand, if each immune serum acted in the same manner with the antigens it would tend to show that while these organisms differ morphologically and biologically in some particulars yet essentially they are the same. The object of this study was as follows:

"To determine the possibility of differentiating the various members of the diphtheria group of organisms by complement-fixation reactions, and particularly to determine the relation of Hofmann's bacillus, also termed the pseudo-diphtheria bacillus, to the true diphtheria organism."

Eight cultures, representing different types of bacilli according to Wesbrook's classification, were selected from different sources. These included one culture fulfilling the morphological, biological, and pathogenic characteristics of Hofmann's bacillus. The types were recorded after the bacilli were secured in pure culture and subcultured several times on serum media. Smears of these cul-

× Arck. Inter, Med., 1912, 9, p. 220. 
tures were made after incubating for 24 hours at $35^{\circ} \mathrm{C}$. and stained with dilute carbol-fuchsin. The following table gives the source, type, sugar reactions, and results of virulence tests:

TABLE I.

\begin{tabular}{|c|c|c|c|c|c|c|c|c|c|c|c|c|c|}
\hline \multirow{2}{*}{ 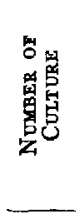 } & \multirow[b]{2}{*}{$\begin{array}{l}\text { SOURCE of } \\
\text { CULTURE }\end{array}$} & \multirow[b]{2}{*}{$\begin{array}{l}\text { Type OF } \\
\text { BACILLUS }\end{array}$} & \multicolumn{7}{|c|}{ Sugar Tests } & \multicolumn{3}{|c|}{$\begin{array}{c}\text { InOculation } \\
\text { TESTS }\end{array}$} & \multirow[b]{2}{*}{ Result } \\
\hline & & & 总 & $\mid \begin{array}{l}\text { 总 } \\
\text { 苞 }\end{array}$ & 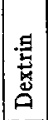 & 总 & 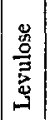 & 萼 & 音 & 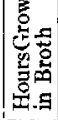 & 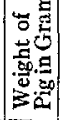 & 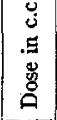 & \\
\hline $\begin{array}{l}2 \ldots \ldots \\
3 \ldots \ldots \\
4 \ldots \ldots\end{array}$ & $\begin{array}{l}\text { Clinical diphtheria } \\
\text { Healthy penis } \\
\text { Park's bac. No. } 8 \\
\text { Throat conval. }\end{array}$ & $\underset{\text { Granular }}{\mathrm{B} \text { and } \mathrm{C}}$ & $\bar{z}$ & $\frac{t}{f}$ & $\frac{+}{+}$ & $\bar{z}$ & $\frac{?}{?}$ & $\begin{array}{l}+ \\
? \\
+\end{array}$ & $\overline{-}$ & $\begin{array}{l}72 \\
72 \\
72\end{array}$ & $\begin{array}{l}260 \\
282 \\
274\end{array}$ & $\begin{array}{l}x .30 \\
\mathrm{I} .4 \\
\mathrm{x} .3\end{array}$ & $\begin{array}{l}\text { Died } \\
\text { Negative } \\
\text { Died }\end{array}$ \\
\hline & $\begin{array}{l}\text { diphtheria } \\
\text { Otitis media } \\
\text { Nasal diphtheria } \\
\text { Throat carrier case } \\
\text { Normal nose }\end{array}$ & $\begin{array}{c}\mathrm{C}_{\mathrm{C}_{z}} \\
\mathrm{D}_{z} \\
\mathrm{C}_{z} \\
\text { Hofmann's bacillus }\end{array}$ & $\begin{array}{l}\overline{ \pm} \\
\overline{-}\end{array}$ & $\begin{array}{l} \pm \\
\pm \\
-\end{array}$ & $\begin{array}{l} \pm \\
\pm \\
-\end{array}$ & $\begin{array}{l}\bar{z} \\
\overline{-} \\
-\end{array}$ & $\begin{array}{c}- \\
\overline{+} \\
+ \\
- \\
-\end{array}$ & $\begin{array}{l}\bar{?} \\
\dot{+} \\
+\end{array}$ & $\bar{z}$ & $\begin{array}{l}72 \\
72 \\
72 \\
72 \\
72\end{array}$ & $\begin{array}{l}290 \\
300 \\
284 \\
260 \\
275\end{array}$ & $\begin{array}{l}\text { I.5 } \\
\text { I.5 } \\
\mathrm{I} .4 \\
\mathrm{I} \cdot 3 \\
2.5\end{array}$ & $\begin{array}{l}\text { Negative } \\
\text { Negative } \\
\text { Died } \\
\text { Negative } \\
\text { Negative }\end{array}$ \\
\hline
\end{tabular}

For media Hiss's serum water with I per cent of the various sugars was used for the acid-production tests. Results were recorded after cultures had been grown for five days at $35^{\circ} \mathrm{C}$.

Virulence tests were carried out with standard-weight pigs and cultures for inoculation were grown in I per cent slightly alkaline glucose broth for 72 hours at $35^{\circ} \mathrm{C}$. with tubes slanted. Injections were made in the median line and animals observed for five days after injection for evidences of edema about the site of injection and symptoms of toxemia. Virulent cultures were retested with toxinantitoxin mixture.

Immune sera.-These were prepared by immunizing a series of rabbits with eight to Io weekly intravenous injections of emulsions of bacilli grown on blood serum media. The first injection of each culture was of organisms heated at $52^{\circ} \mathrm{C}$. for one hour - subsequent injections were living cultures in increasing doses. A number of rabbits succumbed before the final sera were secured. No preservatives were added. Sera were kept in the ice-chest and inactivated before use.

Antigens.-These were prepared of the eight cultures as follows:

I. Grown on serum media for 24 hours and then examined by smear for purity of growth. 
2. Washed off with sterile 0.85 per cent $\mathrm{NaCl}$ solution and strained if particles of media broke away.

3. Emulsions thoroughly centrifuged; supernatant fluid poured off and organisms in the bottom of the centrifuge tubes dried in a cold place over calcium chloride and sulfuric acid. After drying, the tubes were paraffined and placed in ice-box until all sera were ready.

4. The dried bacilli were then ground with powdered glass and shaken for 48 hours in 0.85 per cent $\mathrm{NaCl}$ solution containing 5 per cent phenol, using $0.5 \mathrm{gm}$. of bacilli in ro c.c. of the solution.

5. Bacteria were then thrown down in a high-power electric centrifuge and the supernatant fluid stored in dark glass bottles in ice-chest.

Complement.-Pooled serum of two or more guinea-pigs titrated for exact unit with two units of antisheep hemolysin and I c.c. of 5 per cent suspension of washed sheep corpuscles.

Hemolysin.-Antisheep-diluted so that 0.001 c.c. equaled two units.

Corpuscle suspension.-Washed sheep's corpuscles made up in a 5 per cent suspension. One cubic centimeter used in each test. Antigen, immune serum, and complement were mixed and the total volume brought up to 5 c.c. with the addition of 0.85 per cent $\mathrm{NaCl}$ solution. After tests and controls had been incubated for one hour at $37^{\circ} \mathrm{C}$. hemolysin and corpuscles were added. Reincubated for two hours and then placed in refrigerator for 20 hours, when results were noted.

Antigen and immune sera are designated according to the corresponding number of culture. Thus antigen and immune serum No. I were made from culture No. I and so on.

Antigenic and anti-complementary values of the eight antigens were then determined as shown in Table 2.

The antigen proved fairly satisfactory and 0.08 c.c. was determined as the antigenic unit, although with sera 4 and 7 the degree of complement-fixation was slight but definite.

We were now ready for the most important part of the study, namely, to determine if the antigens would fix complement equally well with all of the different immune sera, or, conversely, if the 
TABLE 2.

Fixing and Anti-Complementary Power of Antigens.

\begin{tabular}{|c|c|c|c|c|c|c|c|c|c|}
\hline $\begin{array}{l}\text { Normal } \\
\text { Serum c.c. }\end{array}$ & $\begin{array}{l}\text { Amount of } \\
\text { Antigen c.c. }\end{array}$ & $\begin{array}{c}\text { Antigen } \\
\text { No. } r\end{array}$ & $\begin{array}{l}\text { Antigen } \\
\text { No. } 2\end{array}$ & $\begin{array}{l}\text { Antigen } \\
\text { No. } 3\end{array}$ & $\begin{array}{c}\text { Antigen } \\
\text { No. } 4\end{array}$ & $\begin{array}{l}\text { Antigen } \\
\text { No. } 5\end{array}$ & $\begin{array}{l}\text { Antigen } \\
\text { No. } 6\end{array}$ & $\begin{array}{c}\text { Antigen } \\
\text { No. } 7\end{array}$ & $\begin{array}{c}\text { Antigen } \\
\text { No. } 8\end{array}$ \\
\hline $\begin{array}{c}0.2 \\
0.2 \\
0.2 \\
0.2 \\
0.2 \\
0.2 \\
0.2 \\
0.2 \\
\text { Immune } \\
\text { Serum c.c. } \\
0.2 \\
0.2 \\
0.2 \\
0.2 \\
0.2 \\
0.2 \\
0.2 \\
0.2\end{array}$ & $\begin{array}{l}0.04 \\
0.06 \\
0.08 \\
0.1 \\
0.15 \\
0.18 \\
0.2 \\
0.3\end{array}$ & \begin{tabular}{|c} 
C.H. \\
C.H. \\
C.H. \\
C.H. \\
C.H. \\
S.I.H. \\
M.I.H. \\
I.H.
\end{tabular} & $\begin{array}{l}\text { M.I.H. } \\
\text { I.H. } \\
\text { I.H. } \\
\text { I.H. } \\
\text { I.H. } \\
\text { I.H. } \\
\text { I.H. } \\
\text { I.H. }\end{array}$ & $\begin{array}{l}\text { S.I.H. } \\
\text { S.I.H. } \\
\text { M.I.H. } \\
\text { I.H. } \\
\text { I.H. } \\
\text { I.H. } \\
\text { I.H. } \\
\text { I.H. }\end{array}$ & $\begin{array}{l}\text { C.H. } \\
\text { C.H. } \\
\text { C.H. } \\
\text { C.H. } \\
\text { C.H. } \\
\text { C.H. } \\
\text { S.I.H. } \\
\text { M.I.H. }\end{array}$ & $\begin{array}{l}\text { C.H. } \\
\text { C.H. } \\
\text { C.H. } \\
\text { C.H. } \\
\text { S.I.H. } \\
\text { S.I.H. } \\
\text { M.I.H. } \\
\text { 1.H. }\end{array}$ & $\begin{array}{l}\text { M.I.H. } \\
\text { I.H. } \\
\text { I.H. } \\
\text { I.H. } \\
\text { I.H. } \\
\text { I.H. } \\
\text { I.H. } \\
\text { I.H. }\end{array}$ & $\begin{array}{l}\text { C.H. } \\
\text { C.H. } \\
\text { C.H. } \\
\text { S.I.H. } \\
\text { S.I.H. } \\
\text { M.I.H. } \\
\text { I.H. } \\
\text { I.H. }\end{array}$ & $\begin{array}{l}\text { C.H. } \\
\text { C.H. } \\
\text { C.H. } \\
\text { S.1.H. } \\
\text { M.I.H. } \\
\text { I.H. } \\
\text { I.H. } \\
\text { I.H. }\end{array}$ \\
\hline
\end{tabular}

*C.H. $=$ complete hemolysis; S.I.H. $=$ slight inhibition of hemolysis; M.1.H. =marked inhibition of hemolysis; I.H. =complete inhibition of hemolysis.

sera with the different antigens would fix complement. The following tables give the results after trying each serum with all of the antigens:

TABLE 3.

IMMUNe Serum No. I.

\begin{tabular}{c|c|c|c|c|c|c|c|c|c}
\hline $\begin{array}{c}\text { Immune } \\
\text { Serum c.c. }\end{array}$ & $\begin{array}{c}\text { Amount of } \\
\text { Antigen c.c. }\end{array}$ & $\begin{array}{c}\text { Antigen } \\
\text { No. I }\end{array}$ & $\begin{array}{c}\text { Antigen } \\
\text { No. 2 }\end{array}$ & $\begin{array}{c}\text { Antigen } \\
\text { No. 3 }\end{array}$ & $\begin{array}{c}\text { Antigen } \\
\text { No. 4 }\end{array}$ & $\begin{array}{c}\text { Antigen } \\
\text { No. 5 }\end{array}$ & $\begin{array}{c}\text { Antigen } \\
\text { No.6 }\end{array}$ & $\begin{array}{c}\text { Antigen } \\
\text { No. 7 }\end{array}$ & $\begin{array}{c}\text { Antigen } \\
\text { No. 8 }\end{array}$ \\
\hline 0.04 & 0.08 & C.H. & C.H. & C.H. & C.H. & C.H. & C.H. & C.H. & C.H. \\
0.06 & 0.08 & S.I.H. & S.I.H. & C.H. & C.H. & C.H. & C.H. & C.H. & C.H. \\
0.08 & 0.08 & M.I.H. & M.I.H. & S.I.H. & C.H. & S.I.H. & C.H. & C.H. & S.l.H. \\
0.1 & 0.08 & I.H. & I.H. & M.I.H. & S.I.H. & S.l.H. & S.l.H. & S.I.H. & M.I.H. \\
0.15 & 0.08 & I.H. & I.H. & I.H. & S.I.H. & M.H.H. & S.I.H. & S.I.H. & I.H. \\
0.18 & 0.08 & I.H. & I.H. & I.H. & M.I.H. & I.H. & M.I.H. & M.I.H. & I.H. \\
0.2 & 0.08 & I.H. & I.H. & I.H. & M.I.H. & I.H. & I.H. & M.I.H. & I.H. \\
\hline
\end{tabular}

TABLE 4.

IMRUNe SeruM No. 2.

\begin{tabular}{|c|c|c|c|c|c|c|c|c|c|}
\hline $\begin{array}{l}\text { Immune } \\
\text { Serum c.c. }\end{array}$ & $\begin{array}{l}\text { Amount of } \\
\text { Antigen c.c. }\end{array}$ & $\begin{array}{c}\text { Antigen } \\
\text { No. I }\end{array}$ & $\begin{array}{c}\text { Antigen } \\
\text { No. } 2\end{array}$ & $\begin{array}{c}\text { Antigen } \\
\text { No. } 3\end{array}$ & $\begin{array}{l}\text { Antigen } \\
\text { No. } 4\end{array}$ & $\begin{array}{c}\text { Antigen } \\
\text { No. } 5\end{array}$ & $\begin{array}{c}\text { Antigen } \\
\text { No. } 6\end{array}$ & $\begin{array}{c}\text { Antigen } \\
\text { No. } 7\end{array}$ & $\begin{array}{c}\text { Antigen } \\
\text { No. } 8\end{array}$ \\
\hline $\begin{array}{l}0.04 \\
0.06 \\
0.08 \\
0.1 \\
0.15 \\
0.18 \\
0.2\end{array}$ & $\begin{array}{l}0.08 \\
0.08 \\
0.08 \\
0.08 \\
0.08 \\
0.08 \\
0.08\end{array}$ & $\begin{array}{c}\text { C.H. } \\
\text { S.I.H. } \\
\text { M.I.H. } \\
\text { I.H. } \\
\text { I.H. } \\
\text { I.H. } \\
\text { I.H. }\end{array}$ & $\begin{array}{l}\text { M.I.H. } \\
\text { M.I.H. } \\
\text { I.H. } \\
\text { I.H. } \\
\text { I.H. } \\
\text { I.H. } \\
\text { I.H. }\end{array}$ & $\begin{array}{c}\text { C.H. } \\
\text { C.H. } \\
\text { S.I.H. } \\
\text { M.I.H. } \\
\text { I.H. } \\
\text { I.H. } \\
\text { I.H. }\end{array}$ & $\begin{array}{l}\text { C.H. } \\
\text { C.H. } \\
\text { C.H. } \\
\text { S.I.H. } \\
\text { M.I.H. } \\
\text { I.H. } \\
\text { I.H. }\end{array}$ & $\begin{array}{l}\text { M.I.H. } \\
\text { M.I.H. } \\
\text { I.H. } \\
\text { I.H. } \\
\text { I.H. } \\
\text { I.H. } \\
\text { I.H. }\end{array}$ & $\begin{array}{c}\text { C.H. } \\
\text { C.H. } \\
\text { C.H. } \\
\text { C.H. } \\
\text { M.I.H. } \\
\text { I.H. } \\
\text { I.H. }\end{array}$ & $\begin{array}{c}\text { C.H. } \\
\text { C.H. } \\
\text { C.H. } \\
\text { S.I.H. } \\
\text { M.I.H. } \\
\text { I.H. } \\
\text { I.H. }\end{array}$ & $\begin{array}{l}\text { I.H. } \\
\text { I.H. } \\
\text { I.H. } \\
\text { I.H. } \\
\text { I.H. } \\
\text { I.H. } \\
\text { I.H. }\end{array}$ \\
\hline
\end{tabular}


TABLE 5-

IMMUNe Serom No. 3 .

\begin{tabular}{|c|c|c|c|c|c|c|c|c|c|}
\hline $\begin{array}{l}\text { Immune } \\
\text { Serum c.c. }\end{array}$ & $\begin{array}{l}\text { Amount of } \\
\text { Antigen c.c. }\end{array}$ & $\begin{array}{c}\text { Antigen } \\
\text { No. I }\end{array}$ & $\begin{array}{c}\text { Antigen } \\
\text { No. } 2\end{array}$ & $\begin{array}{c}\text { Antigen } \\
\text { No. } 3\end{array}$ & $\begin{array}{c}\text { Antigen } \\
\text { No. } 4\end{array}$ & $\begin{array}{l}\text { Antigen } \\
\text { No. } 5\end{array}$ & $\begin{array}{c}\text { Antigen } \\
\text { No. } 6\end{array}$ & $\begin{array}{c}\text { Antigen } \\
\text { No. } 7\end{array}$ & $\begin{array}{l}\text { Antigen } \\
\text { No. } 8\end{array}$ \\
\hline $\begin{array}{l}0.04 \\
0.06 \\
0.08 \\
0.1 \\
0.15 \\
0.18 \\
0.2\end{array}$ & $\begin{array}{l}0.08 \\
0.08 \\
0.08 \\
0.08 \\
0.08 \\
0.08 \\
0.08\end{array}$ & $\begin{array}{l}\text { C.H. } \\
\text { C.H. } \\
\text { C.H. } \\
\text { C.H. } \\
\text { C.H. } \\
\text { C.H. } \\
\text { S.I.H. }\end{array}$ & $\begin{array}{l}\text { C.H. } \\
\text { C.H. } \\
\text { C.H. } \\
\text { C.H. } \\
\text { C.H. } \\
\text { S.I.H. } \\
\text { M.I.H. }\end{array}$ & $\begin{array}{c}\text { C.H. } \\
\text { C.H. } \\
\text { C.H. } \\
\text { C.H. } \\
\text { C.H. } \\
\text { M.I.H. } \\
\text { I.H. }\end{array}$ & $\begin{array}{c}\text { C.H. } \\
\text { C.H. } \\
\text { C.H. } \\
\text { C.H. } \\
\text { C.H. } \\
\text { S.I.H. } \\
\text { M.I.H. }\end{array}$ & $\begin{array}{c}\text { C.H. } \\
\text { C.H. } \\
\text { C.H. } \\
\text { C.H. } \\
\text { S.I.H. } \\
\text { M.I.H. } \\
\text { I.H. }\end{array}$ & $\begin{array}{l}\text { C.H. } \\
\text { C.H. } \\
\text { C.H. } \\
\text { C.H. } \\
\text { C.H. } \\
\text { C.H. } \\
\text { S.I.H. }\end{array}$ & $\begin{array}{l}\text { C.H. } \\
\text { C.H. } \\
\text { C.H. } \\
\text { S.I.H. } \\
\text { M.I.H. } \\
\text { M.I.H. } \\
\text { I.H. }\end{array}$ & $\begin{array}{l}\text { C.H. } \\
\text { C.H. } \\
\text { C..H. } \\
\text { C.H. } \\
\text { C.H. } \\
\text { S.I.H. } \\
\text { M.I.H. }\end{array}$ \\
\hline
\end{tabular}

TABLE 6.

IMMUNe Serum No. 4.

\begin{tabular}{|c|c|c|c|c|c|c|c|c|c|}
\hline $\begin{array}{l}\text { Immune } \\
\text { Serum c.c. }\end{array}$ & $\begin{array}{l}\text { Amount of } \\
\text { Antigen c.c. }\end{array}$ & $\begin{array}{c}\text { Antigen } \\
\text { No. I }\end{array}$ & $\begin{array}{c}\text { Antigen } \\
\text { No. 2 }\end{array}$ & $\begin{array}{l}\text { Antigen } \\
\text { No. } 3\end{array}$ & $\begin{array}{c}\text { Antigen } \\
\text { No. } 4\end{array}$ & $\begin{array}{c}\text { Antigen } \\
\text { No. } 5\end{array}$ & $\begin{array}{c}\text { Antigen } \\
\text { No. } 6\end{array}$ & $\begin{array}{l}\text { Antigen } \\
\text { No. } 7\end{array}$ & $\begin{array}{c}\text { Antigen } \\
\text { No. } 8\end{array}$ \\
\hline $\begin{array}{l}0.04 \\
0.06 \\
0.08 \\
0.1 \\
0.15 \\
0.18 \\
0.2\end{array}$ & $\begin{array}{l}0.08 \\
0.08 \\
0.08 \\
0.08 \\
0.08 \\
0.08 \\
0.08\end{array}$ & $\begin{array}{c}\text { C.H. } \\
\text { C.H. } \\
\text { C.H. } \\
\text { C.H. } \\
\text { S.I.H. } \\
\text { M.I.H. } \\
\text { I.H. }\end{array}$ & $\begin{array}{l}\text { C.H. } \\
\text { C.H. } \\
\text { C.H. } \\
\text { S.I.H. } \\
\text { M.I.H. } \\
\text { I.H. } \\
\text { I.H. }\end{array}$ & $\begin{array}{c}\text { C.H. } \\
\text { C.H. } \\
\text { C.H. } \\
\text { C.H. } \\
\text { S.I.H. } \\
\text { M.I.H. } \\
\text { I.H. }\end{array}$ & $\begin{array}{c}\text { C.H. } \\
\text { C.H. } \\
\text { S.I.H. } \\
\text { M.1.H. } \\
\text { I.H. } \\
\text { 1.H. } \\
\text { I.H. }\end{array}$ & $\begin{array}{l}\text { I.H. } \\
\text { I.H. } \\
\text { I.H. } \\
\text { I.H. } \\
\text { I.H. } \\
\text { I.H. } \\
\text { I.H. }\end{array}$ & $\begin{array}{c}\text { M.I.H. } \\
\text { I.H. } \\
\text { I.H. } \\
\text { I.H. } \\
\text { I.H. } \\
\text { I.H. } \\
\text { I.H. }\end{array}$ & $\begin{array}{l}\text { I.H. } \\
\text { I.H. } \\
\cdots \cdots \\
\cdots \cdots \\
\cdots \cdots \\
\cdots \cdots\end{array}$ & $\begin{array}{l}\ldots \\
\ldots \ldots \\
\ldots \ldots \\
\ldots \ldots \\
\ldots\end{array}$ \\
\hline
\end{tabular}

TABLE 7 .

IMARUNe SERUM No. 5.

\begin{tabular}{|c|c|c|c|c|c|c|c|c|c|}
\hline $\begin{array}{l}\text { Immune } \\
\text { Serum c.c. }\end{array}$ & $\begin{array}{l}\text { Amount of } \\
\text { Antigen c.c. }\end{array}$ & $\begin{array}{c}\text { Antigen } \\
\text { No. I }\end{array}$ & $\begin{array}{c}\text { Antigen } \\
\text { No. } 2\end{array}$ & $\begin{array}{c}\text { Antigen } \\
\text { No. } 3\end{array}$ & $\begin{array}{c}\text { Antigen } \\
\text { No. } 4\end{array}$ & $\begin{array}{c}\text { Antigen } \\
\text { No. } 5\end{array}$ & $\begin{array}{c}\text { Antigen } \\
\text { No. } 6\end{array}$ & $\begin{array}{c}\text { Antigen } \\
\text { No. } 7\end{array}$ & $\begin{array}{c}\text { Antigen } \\
\text { No. } 8\end{array}$ \\
\hline $\begin{array}{l}0.04 \\
0.06 \\
0.08 \\
0.1 \\
0.15 \\
0.18 \\
0.2\end{array}$ & $\begin{array}{l}0.08 \\
0.08 \\
0.08 \\
0.08 \\
0.08 \\
0.08 \\
0.08\end{array}$ & $\begin{array}{l}\text { C.H. } \\
\text { C.H. } \\
\text { C.H. } \\
\text { C.H. } \\
\text { C.H. } \\
\text { S.I.H. } \\
\text { S.I.H. }\end{array}$ & $\begin{array}{l}\text { C.H. } \\
\text { C.H. } \\
\text { C.H. } \\
\text { C.H. } \\
\text { C.H. } \\
\text { C.H. } \\
\text { S.I.H. }\end{array}$ & $\begin{array}{l}\text { C.H. } \\
\text { C.H. } \\
\text { C.H. } \\
\text { C.H. } \\
\text { C.H. } \\
\text { C.H. } \\
\text { S.I.H. }\end{array}$ & $\begin{array}{c}\text { C.H. } \\
\text { C.H. } \\
\text { C.H. } \\
\text { C.H. } \\
\text { C.H. } \\
\text { M.I.H. } \\
\text { I.H. }\end{array}$ & $\begin{array}{l}\text { C.H. } \\
\text { C.H. } \\
\text { S.I.H. } \\
\text { M.I.H. } \\
\text { M.I.H. } \\
\text { I.H. } \\
\text { I.H. }\end{array}$ & $\begin{array}{c}\text { C.H. } \\
\text { C.H. } \\
\text { C.H. } \\
\text { C.H. } \\
\text { S.I.H. } \\
\text { M.I.H. } \\
\text { I.H. }\end{array}$ & $\begin{array}{c}\text { C.H. } \\
\text { C.H. } \\
\text { C.H. } \\
\text { C.H. } \\
\text { S.I.H. } \\
\text { S.I.H. } \\
\text { M.I.H. }\end{array}$ & $\begin{array}{l}\text { C.H. } \\
\text { C.H. } \\
\text { C.H. } \\
\text { C.H. } \\
\text { C.H. } \\
\text { C.H. } \\
\text { S.I.H. }\end{array}$ \\
\hline
\end{tabular}

TABLE 8.

TMMUNE SERUM NO. 6 .

\begin{tabular}{|c|c|c|c|c|c|c|c|c|c|}
\hline $\begin{array}{l}\text { Immune } \\
\text { Serum c.c. }\end{array}$ & $\begin{array}{l}\text { Amount of } \\
\text { Antigen c.c. }\end{array}$ & $\begin{array}{c}\text { Antigen } \\
\text { No. I }\end{array}$ & $\begin{array}{c}\text { Antigen } \\
\text { No. } 2\end{array}$ & $\begin{array}{c}\text { Antigen } \\
\text { No. } 3\end{array}$ & $\begin{array}{c}\text { Antigen } \\
\text { No. } 4\end{array}$ & $\begin{array}{c}\text { Antigen } \\
\text { No. } 5\end{array}$ & $\begin{array}{c}\text { Antigen } \\
\text { No. } 6\end{array}$ & $\begin{array}{c}\text { Antigen } \\
\text { No. } 7\end{array}$ & $\begin{array}{c}\text { Antigen } \\
\text { No. } 8\end{array}$ \\
\hline $\begin{array}{l}0.04 \\
0.06 \\
0.08 \\
0.1 \\
0.15 \\
0.18 \\
0.2\end{array}$ & $\begin{array}{l}0.08 \\
0.08 \\
0.08 \\
0.08 \\
0.08 \\
0.08 \\
0.08\end{array}$ & $\begin{array}{l}\text { C.H. } \\
\text { C.H. } \\
\text { C.H. } \\
\text { C.H. } \\
\text { C.H. } \\
\text { S.1.H. } \\
\text { M.I.H. }\end{array}$ & $\begin{array}{l}\text { C.H. } \\
\text { C.H. } \\
\text { C.H. } \\
\text { C.H. } \\
\text { C.H. } \\
\text { C.H. } \\
\text { S.I.H. }\end{array}$ & $\begin{array}{l}\text { C.H. } \\
\text { C.H. } \\
\text { C.H. } \\
\text { C.H. } \\
\text { C.H. } \\
\text { C.H. } \\
\text { S.1.H. }\end{array}$ & $\begin{array}{c}\text { C.H. } \\
\text { C.H. } \\
\text { C.H. } \\
\text { C.H. } \\
\text { C.H. } \\
\text { S.I.H. } \\
\text { M.I.H. }\end{array}$ & $\begin{array}{l}\text { C.H. } \\
\text { C.H. } \\
\text { S.I.H. } \\
\text { S.I.H. } \\
\text { M.I.H. } \\
\text { l.H. } \\
\text { I.H. }\end{array}$ & $\begin{array}{l}\text { C.H. } \\
\text { C.H. } \\
\text { C.H. } \\
\text { S.I.H. } \\
\text { S.l.H. } \\
\text { M.I.H. } \\
\text { I.H. }\end{array}$ & $\begin{array}{l}\text { C.H. } \\
\text { C.H. } \\
\text { C.H. } \\
\text { C.H. } \\
\text { C.H. } \\
\text { C.H. } \\
\text { S.I.H. }\end{array}$ & $\begin{array}{c}\text { S.I.H. } \\
\text { M.I.H. } \\
\text { I.H. } \\
\text { I.H. } \\
\text { I.H. } \\
\text { I.H. } \\
\text { I.H. }\end{array}$ \\
\hline
\end{tabular}


TABLE 9 .

IMMUNe SERUM No. 7.

\begin{tabular}{|c|c|c|c|c|c|c|c|c|c|}
\hline $\begin{array}{l}\text { Immune } \\
\text { Serum c.c. }\end{array}$ & $\begin{array}{l}\text { Amount of } \\
\text { Antigen c.c. }\end{array}$ & $\begin{array}{c}\text { Antigen } \\
\text { No. I }\end{array}$ & $\begin{array}{c}\text { Antigen } \\
\text { No. } 2\end{array}$ & $\begin{array}{c}\text { Antigen } \\
\text { No. } 3\end{array}$ & $\begin{array}{c}\text { Antigen } \\
\text { No. } 4\end{array}$ & $\begin{array}{c}\text { Antigen } \\
\text { No. } 5\end{array}$ & $\begin{array}{c}\text { Antigen } \\
\text { No. } 6\end{array}$ & $\begin{array}{c}\text { Antigen } \\
\text { No. } 7\end{array}$ & $\begin{array}{c}\text { Antigen } \\
\text { No. } 8\end{array}$ \\
\hline $\begin{array}{l}0.04 \\
0.06 \\
0.08 \\
0.1 \\
0.15 \\
0.18 \\
0.2\end{array}$ & $\begin{array}{l}0.08 \\
0.08 \\
0.08 \\
0.08 \\
0.08 \\
0.08 \\
0.08\end{array}$ & $\begin{array}{l}\text { C.H. } \\
\text { C.H. } \\
\text { C.H. } \\
\text { C.H. } \\
\text { C.H. } \\
\text { C.H. } \\
\text { C.H. }\end{array}$ & $\begin{array}{l}\text { C.H. } \\
\text { C.H. } \\
\text { C.H. } \\
\text { C.H. } \\
\text { C.H. } \\
\text { S.I.H. } \\
\text { S.I.H. }\end{array}$ & $\begin{array}{l}\text { C.H. } \\
\text { C.H. } \\
\text { C.H. } \\
\text { C.H. } \\
\text { C.H. } \\
\text { C.H. } \\
\text { S.I.H. }\end{array}$ & $\begin{array}{l}\text { C.H. } \\
\text { C.H. } \\
\text { C.H. } \\
\text { C.H. } \\
\text { C.H. } \\
\text { C.H. } \\
\text { C.H. }\end{array}$ & $\begin{array}{l}\text { C.H. } \\
\text { C.H. } \\
\text { S.I.H. } \\
\text { S.I.H. } \\
\text { M.I.H. } \\
\text { M.I.H. } \\
\text { I.H. }\end{array}$ & $\begin{array}{l}\text { C.H. } \\
\text { C.H. } \\
\text { C.H. } \\
\text { C.H. } \\
\text { C.H. } \\
\text { C.H. } \\
\text { C.H. }\end{array}$ & $\begin{array}{l}\text { C.H. } \\
\text { C.H. } \\
\text { C.H. } \\
\text { C.H. } \\
\text { C.H. } \\
\text { C.H. } \\
\text { S.I.H. }\end{array}$ & $\begin{array}{l}\text { C.H. } \\
\text { C.H. } \\
\text { C.H. } \\
\text { S.I.H. } \\
\text { S.I.H. } \\
\text { M.I.H. } \\
\text { M.I.H. }\end{array}$ \\
\hline
\end{tabular}

TABLE Io.

IMMUNE SERUM No. 8.

\begin{tabular}{|c|c|c|c|c|c|c|c|c|c|}
\hline $\begin{array}{l}\text { Immune } \\
\text { Serum c.c. }\end{array}$ & $\begin{array}{l}\text { Amount of } \\
\text { Antigen c.c. }\end{array}$ & $\underset{\text { No. I }}{\text { Antigen }}$ & $\begin{array}{c}\text { Antigen } \\
\text { No. } 2\end{array}$ & $\begin{array}{c}\text { Antigen } \\
\text { No. } 3\end{array}$ & $\begin{array}{c}\text { Antigen } \\
\text { No. } 4\end{array}$ & $\begin{array}{c}\text { Antigen } \\
\text { No. } 5\end{array}$ & $\begin{array}{c}\text { Antigen } \\
\text { No. } 6\end{array}$ & $\begin{array}{l}\text { Antigen } \\
\text { No. } 7\end{array}$ & $\begin{array}{l}\text { Antigen } \\
\text { No. } 8\end{array}$ \\
\hline $\begin{array}{l}0.04 \\
0.06 \\
0.08 \\
0.1 \\
0.15 \\
9.18 \\
0.2\end{array}$ & $\begin{array}{l}0.08 \\
0.08 \\
0.08 \\
0.08 \\
0.08 \\
0.08 \\
0.08\end{array}$ & $\begin{array}{c}\text { C.H. } \\
\text { C.H. } \\
\text { C.H. } \\
\text { C.H. } \\
\text { S.I.H. } \\
\text { M.I.H. } \\
\text { M.I.H. }\end{array}$ & $\begin{array}{c}\text { C.H. } \\
\text { C.H. } \\
\text { C.H. } \\
\text { C.H. } \\
\text { C.H. } \\
\text { S.I.H. } \\
\text { M.1.H. }\end{array}$ & $\begin{array}{c}\text { C.H. } \\
\text { C.H. } \\
\text { C.H. } \\
\text { C.H. } \\
\text { S.I.H. } \\
\text { S.I.H. } \\
\text { M.I.H. }\end{array}$ & $\begin{array}{l}\text { C.H. } \\
\text { C.I. } \\
\text { C.H. } \\
\text { C.H. } \\
\text { C.H. } \\
\text { S.I.H. } \\
\text { S.I.H. }\end{array}$ & $\begin{array}{l}\text { I.H. } \\
\text { I.H. } \\
\mathbf{l} . \mathbf{H} . \\
\ldots \ldots \\
\ldots \\
\ldots \\
\ldots\end{array}$ & $\begin{array}{c}\text { C.H. } \\
\text { C.H. } \\
\text { C.H. } \\
\text { C.H. } \\
\text { S.I.H. } \\
\text { M.I.H. } \\
\text { M.I.H. }\end{array}$ & $\begin{array}{c}\text { C.H. } \\
\text { C.H. } \\
\text { C.H. } \\
\text { C.H. } \\
\text { C.H. } \\
\text { S.I.H. } \\
\text { M.I.H. }\end{array}$ & $\begin{array}{l}\text { I.H. } \\
\text { I.H. } \\
\ldots \ldots \\
\ldots \\
\ldots \\
\ldots \\
\ldots\end{array}$ \\
\hline
\end{tabular}

Following these experiments the sera from seven cases of diphtheria were tried with the eight different antigens. These sera were collected four days previous to setting up the tests; sera were inactivated by heating to $55^{\circ} \mathrm{C}$. for 25 minutes.

CASE I.

J. B.; age 6 years; tonsillar diphtheria due to diphtheria bacillus, type C. Patient had received 5,000 units of antitoxin four days ago:

\begin{tabular}{c|c|c|c|c|c|c|c|c|c}
\hline \hline $\begin{array}{c}\text { Serum } \\
\text { c.c. }\end{array}$ & $\begin{array}{c}\text { Amount of } \\
\text { Antigen c.c. }\end{array}$ & $\begin{array}{c}\text { Antigen } \\
\text { No. I }\end{array}$ & $\begin{array}{c}\text { Antigen } \\
\text { No. 2 }\end{array}$ & $\begin{array}{c}\text { Antigen } \\
\text { No. 3 }\end{array}$ & $\begin{array}{c}\text { Antigen } \\
\text { No. 4 }\end{array}$ & $\begin{array}{c}\text { Antigen } \\
\text { No. 5 }\end{array}$ & $\begin{array}{c}\text { Antigen } \\
\text { No. 6 }\end{array}$ & $\begin{array}{c}\text { Antigen } \\
\text { No. 7 }\end{array}$ & $\begin{array}{c}\text { Antigen } \\
\text { No. 8 }\end{array}$ \\
\hline $\begin{array}{l}0.05 \\
0.1\end{array}$ & 0.08 & C.H. & C.H. & C.H. & C.H. & C.H. & C.H. & C.H. & C.H. \\
0.2 & 0.08 & S.I.H. & C.H. & S.I.H. & C.H. & C.H. & C.H. & C.H. & C.H. \\
\hline
\end{tabular}

CASE 2.

H. R.; age 4 years and 9 months; developed tonsillar diphtheria three days ago; diphtheria bacillus, type $\mathrm{C}$; received 5,000 units of antitoxin three days ago:

\begin{tabular}{|c|c|c|c|c|c|c|c|c|c|}
\hline $\begin{array}{l}\text { Serum } \\
\text { c.c. }\end{array}$ & $\begin{array}{l}\text { Amount of } \\
\text { Antigen c.c. }\end{array}$ & $\begin{array}{c}\text { Antigen } \\
\text { No. I }\end{array}$ & $\begin{array}{c}\text { Antigen } \\
\text { No. 2 }\end{array}$ & $\begin{array}{c}\text { Antigen } \\
\text { No. } 3\end{array}$ & $\begin{array}{c}\text { Antigen } \\
\text { No. } 4 \\
\end{array}$ & $\begin{array}{c}\text { Antigen } \\
\text { No. } 5\end{array}$ & $\begin{array}{c}\text { Antigen } \\
\text { No. } 6\end{array}$ & $\begin{array}{l}\text { Antigen } \\
\text { No. } 7\end{array}$ & $\begin{array}{c}\text { Antigen } \\
\text { No. } 8\end{array}$ \\
\hline $\begin{array}{l}0.05 \\
0 . I \\
0.2\end{array}$ & $\begin{array}{l}0.08 \\
0.08 \\
0.08\end{array}$ & $\begin{array}{l}\text { C.H. } \\
\text { S.I.H. } \\
\text { M.I.H. }\end{array}$ & $\begin{array}{l}\text { C.H. } \\
\text { S.I.H. } \\
\text { M.I.H. }\end{array}$ & $\begin{array}{l}\text { S.I.H. } \\
\text { M.I.H. } \\
\text { I.H. }\end{array}$ & $\begin{array}{l}\text { C.H. } \\
\text { S.I.H. } \\
\text { s.t.H. }\end{array}$ & $\begin{array}{l}\text { C.H. } \\
\text { C.H. } \\
\text { C.H. }\end{array}$ & $\begin{array}{l}\text { C.H. } \\
\text { C.H. } \\
\text { C.H. }\end{array}$ & $\begin{array}{l}\text { C.H. } \\
\text { S.I.H. } \\
\text { S.Y.H. }\end{array}$ & $\begin{array}{l}\text { C.H. } \\
\text { C.H. } \\
\text { C.H. }\end{array}$ \\
\hline
\end{tabular}


CASE 3 .

D. R.; age 12 years; developed tonsillar diphtheria six days ago, type C. Received unknown quantity of antitoxin previous to admittance to hospital.

\begin{tabular}{|c|c|c|c|c|c|c|c|c|c|}
\hline $\begin{array}{l}\text { Immune } \\
\text { Serum c.c. }\end{array}$ & $\begin{array}{l}\text { Amount of } \\
\text { Antigen c.c. }\end{array}$ & $\begin{array}{c}\text { Antigen } \\
\text { No. I }\end{array}$ & $\begin{array}{c}\text { Antigen } \\
\text { No. } 2\end{array}$ & $\begin{array}{c}\text { Antigen } \\
\text { No. } 3\end{array}$ & $\begin{array}{c}\text { Antigen } \\
\text { No. } 4\end{array}$ & $\begin{array}{c}\text { Antigen } \\
\text { No. } 5\end{array}$ & $\begin{array}{c}\text { Antigen } \\
\text { No. } 6\end{array}$ & $\begin{array}{c}\text { Antigen } \\
\text { No. } 7\end{array}$ & $\begin{array}{c}\text { Antigen } \\
\text { No. } 8\end{array}$ \\
\hline $\begin{array}{l}0.05 \\
0.1 \\
0.2\end{array}$ & $\begin{array}{l}0.08 \\
0.08 \\
0.08\end{array}$ & $\begin{array}{c}\text { C.H. } \\
\text { S.I.H. } \\
\text { M.I.H. }\end{array}$ & $\begin{array}{c}\text { C.H. } \\
\text { S.I.H. } \\
\text { M.I.H }\end{array}$ & $\begin{array}{c}\text { C.H. } \\
\text { S.I.H. } \\
\text { M.I.H. }\end{array}$ & $\begin{array}{l}\text { S.I.H. } \\
\text { S.I.H. } \\
\text { M.I.H. }\end{array}$ & $\begin{array}{c}\text { C.H. } \\
\text { S.I.H. } \\
\text { M.I.H. }\end{array}$ & $\begin{array}{c}\text { C.H. } \\
\text { C.H. } \\
\text { S.I.H. }\end{array}$ & $\begin{array}{l}\text { C.H. } \\
\text { C.H. } \\
\text { S.I.H. }\end{array}$ & $\begin{array}{l}\text { C.H. } \\
\text { C.H. } \\
\text { C.H. }\end{array}$ \\
\hline
\end{tabular}

CASE 4 .

F. B.; age $4 \frac{1}{2}$ years; developed tonsillar diphtheria five days ago, type $\mathrm{C}_{2}$ bacillus. Received 4,000 units of antitoxin four days ago.

\begin{tabular}{|c|c|c|c|c|c|c|c|c|c|}
\hline $\begin{array}{l}\text { Serum } \\
\text { c.c. }\end{array}$ & $\begin{array}{l}\text { Amount of } \\
\text { Antigen c.c. }\end{array}$ & $\begin{array}{c}\text { Antigen } \\
\text { No. I }\end{array}$ & $\begin{array}{c}\text { Antigen } \\
\text { No. } 2\end{array}$ & $\begin{array}{c}\text { Antigen } \\
\text { No. }-3\end{array}$ & $\begin{array}{l}\text { Antigen } \\
\text { No. } 4\end{array}$ & $\begin{array}{c}\text { Antigen } \\
\text { No. } 5\end{array}$ & $\begin{array}{c}\text { Antigen } \\
\text { No. } 6\end{array}$ & $\begin{array}{c}\text { Antigen } \\
\text { No. } 7\end{array}$ & $\begin{array}{c}\text { Antigen } \\
\text { No. } 8\end{array}$ \\
\hline $\begin{array}{l}0.05 \\
0.1 \\
0.2\end{array}$ & $\begin{array}{l}0.08 \\
0.08 \\
0.08\end{array}$ & $\begin{array}{l}\text { C.H. } \\
\text { C.H. } \\
\text { C.H. }\end{array}$ & $\begin{array}{l}\text { C.H. } \\
\text { C.H. } \\
\text { S.I.H. }\end{array}$ & $\begin{array}{l}\text { C.H. } \\
\text { C.H. } \\
\text { C.H. }\end{array}$ & $\begin{array}{l}\text { C.H. } \\
\text { C.H. } \\
\text { C.H. }\end{array}$ & $\begin{array}{l}\text { C.H. } \\
\text { C.H. } \\
\text { S.T.H. }\end{array}$ & $\begin{array}{l}\text { C.H. } \\
\text { C.H. } \\
\text { C.H. }\end{array}$ & $\begin{array}{l}\text { C.H. } \\
\text { C.H. } \\
\text { C.H. }\end{array}$ & $\begin{array}{c}\text { C.H. } \\
\text { S.I.H. } \\
\text { M.I.H. }\end{array}$ \\
\hline
\end{tabular}

CASE 5 .

S. S.; age 14 years; devcloped tonsillar diphtheria seven days ago, type C bacillus. Received 10,000 units of antitoxin six days ago:

\begin{tabular}{|c|c|c|c|c|c|c|c|c|c|}
\hline $\begin{array}{l}\text { Serum } \\
\text { c.c. }\end{array}$ & $\begin{array}{l}\text { A mount of } \\
\text { Antigen c.c. }\end{array}$ & $\begin{array}{c}\text { Antigen } \\
\text { No. } x\end{array}$ & $\begin{array}{c}\text { Antigen } \\
\text { No. } 2\end{array}$ & $\begin{array}{c}\text { Antigen } \\
\text { No. } 3\end{array}$ & $\begin{array}{l}\text { Antigen } \\
\text { No. } 4\end{array}$ & $\begin{array}{l}\text { Antigen } \\
\text { No. } 5\end{array}$ & $\begin{array}{c}\text { Antigen } \\
\text { No. } 6\end{array}$ & $\begin{array}{l}\text { Antigen } \\
\text { No. } 7\end{array}$ & $\begin{array}{c}\text { Antigen } \\
\text { No. } 8\end{array}$ \\
\hline $\begin{array}{l}0.05 \\
0.1 \\
0.2\end{array}$ & $\begin{array}{l}0.08 \\
0.08 \\
0.08\end{array}$ & $\begin{array}{l}\text { C.H. } \\
\text { C.H. } \\
\text { C.H. }\end{array}$ & $\begin{array}{l}\text { C.H.H. } \\
\text { C.H. }\end{array}$ & $\begin{array}{l}\text { C.H. } \\
\text { C.H. } \\
\text { s.i.H. }\end{array}$ & $\begin{array}{l}\text { C.H. } \\
\text { C.H. } \\
\text { C.H. }\end{array}$ & $\begin{array}{l}\text { C.H. } \\
\text { C.H. } \\
\text { S.I.H. }\end{array}$ & $\begin{array}{c}\text { C.H. } \\
\text { C.H. } \\
\text { S.I.H. }\end{array}$ & $\begin{array}{l}\text { C.H. } \\
\text { C.H. } \\
\text { C.H. }\end{array}$ & $\begin{array}{l}\text { C.H. } \\
\text { C.H. } \\
\text { C.H. }\end{array}$ \\
\hline
\end{tabular}

CASE 6.

J. K.; age I 2 years; developed tonsillar diphtheria I I days ago, type A bacillus. Received 10,000 units of antitoxin eight days ago:

\begin{tabular}{|c|c|c|c|c|c|c|c|c|c|}
\hline $\begin{array}{l}\text { Serum } \\
\text { c.c. }\end{array}$ & $\begin{array}{l}\text { Amount of } \\
\text { Antigen c.c. }\end{array}$ & $\begin{array}{l}\text { Antigen } \\
\text { No. I }\end{array}$ & $\begin{array}{c}\text { Antigen } \\
\text { No. } 2\end{array}$ & $\begin{array}{c}\text { Antigen } \\
\text { No. 3 }\end{array}$ & $\begin{array}{c}\text { Antigen } \\
\text { No. } 4\end{array}$ & $\begin{array}{c}\text { Antigen } \\
\text { No. } 5\end{array}$ & $\underset{\text { No. } 6}{\text { Antigen }}$ & $\begin{array}{c}\text { Antigen } \\
\text { No, } 7\end{array}$ & $\begin{array}{c}\text { Antigen } \\
\text { No. } 8\end{array}$ \\
\hline $\begin{array}{l}0.05 \\
0.1 \\
0.2\end{array}$ & $\begin{array}{l}0.08 \\
0.08 \\
0.08\end{array}$ & $\begin{array}{l}\text { C.H. } \\
\text { C.H. } \\
\text { C.H. }\end{array}$ & $\begin{array}{l}\text { C.H. } \\
\text { C.H. } \\
\text { C.H. }\end{array}$ & $\begin{array}{l}\text { C.H. } \\
\text { C.H. } \\
\text { C.H. }\end{array}$ & $\begin{array}{c}\text { C.H. } \\
\text { C.H. } \\
\text { S.I.H. }\end{array}$ & $\begin{array}{l}\text { C.H. } \\
\text { S.I.H. } \\
\text { M.I.H. }\end{array}$ & $\begin{array}{l}\text { C.H. } \\
\text { C.H. } \\
\text { C.H. }\end{array}$ & $\begin{array}{l}\text { C.H. } \\
\text { C.H. } \\
\text { C.H. }\end{array}$ & $\begin{array}{l}\text { C.H. } \\
\text { S.I.H. } \\
\text { M.I.H. }\end{array}$ \\
\hline
\end{tabular}

CASE 7 .

J. C.; age 12 years; developed tonsillar diphtheria 14 days ago, type $\mathrm{C}$ bacillus. Received so,ooo units of antitoxin ro days ago:

\begin{tabular}{|c|c|c|c|c|c|c|c|c|c|}
\hline $\begin{array}{l}\text { Serum } \\
\text { c.c. }\end{array}$ & $\begin{array}{l}\text { Amount of } \\
\text { Antigen c.c. }\end{array}$ & $\begin{array}{c}\text { Antigen } \\
\text { No. I }\end{array}$ & $\begin{array}{c}\text { Antigen } \\
\text { No. } 2\end{array}$ & $\begin{array}{c}\text { Antigen } \\
\text { No. } 3\end{array}$ & $\begin{array}{l}\text { Antigen } \\
\text { No. } 4\end{array}$ & $\begin{array}{c}\text { Antigen } \\
\text { No. } 5\end{array}$ & $\begin{array}{c}\text { Antigen } \\
\text { No. } 6\end{array}$ & $\begin{array}{c}\text { Antigen } \\
\text { No. } 7\end{array}$ & $\begin{array}{l}\text { Antigen } \\
\text { No. } 8\end{array}$ \\
\hline $\begin{array}{l}0.05 \\
0.1 \\
0.2\end{array}$ & $\begin{array}{l}0.08 \\
0.08 \\
0.08\end{array}$ & $\begin{array}{l}\text { C. } . H . \\
\text { C.H. } \\
\text { C.H. }\end{array}$ & $\begin{array}{c}\text { C.H. } \\
\text { S.I.H. } \\
\text { M.I.H. }\end{array}$ & $\begin{array}{l}\text { C.H. } \\
\text { C.H. } \\
\text { C.H. }\end{array}$ & $\begin{array}{l}\text { C.H. } \\
\text { C.H. } \\
\text { C.H. }\end{array}$ & $\begin{array}{l}\text { C. } \mathrm{H} . \\
\text { C.H. } \\
\text { C.H. }\end{array}$ & $\begin{array}{l}\text { C.H. } \\
\text { C.H. } \\
\text { C.H. }\end{array}$ & $\begin{array}{l}\text { C.H. } \\
\text { C.H. } \\
\text { C.H. }\end{array}$ & $\begin{array}{l}\text { C.H. } \\
\text { C.H. } \\
\text { C.H. }\end{array}$ \\
\hline
\end{tabular}




\section{RESULTS.}

While a study of the tables shows in a few instances a tendency to specific reaction, yet as a whole the results indicate most conclusively that all the immune sera and antigens acted in quite a similar manner, and, as far as complement-fixation tests are capable of indicating specific relations between immune body and its antigen these reactions show that each of the different types of diphtheria bacilli, including Hofmann's bacillus, are capable of causing the production of similar immune bodies.

\section{CONTROLS.}

That there is developed in the serum of an animal immunized with diphtheria bacilli a body capable of fixing complement only with its homologous antigen is seen in the following two tables where different antigens were used with the various diphtheria immune sera with uniform failure of complement-fixation:

TABLE II.

Antigen of alcoholic extract of fetal syphilitic liver; antigenic for syphilitic antibody in amount of 0.2 c.c. of $1: 3$ dilution; becomes anti-complementary in amounts of 0.9 c.c. (dil. $I: 3$ ).

\begin{tabular}{|c|c|c|c|c|c|c|c|c|c|}
\hline $\begin{array}{c}\text { Serum } \\
\text { c.c. }\end{array}$ & $\begin{array}{l}\text { Amount of } \\
\text { Antigen c.c. }\end{array}$ & $\underset{I}{S e r u m}$ & $\underset{2}{\text { Serum }}$ & $\underset{3}{\text { Serum }}$ & $\begin{array}{c}\text { Serum } \\
4\end{array}$ & $\underset{5}{\text { Serum }}$ & $\underset{6}{\text { Serum }}$ & $\begin{array}{c}\text { Serum } \\
7\end{array}$ & $\underset{8}{\operatorname{Serum}}$ \\
\hline $\begin{array}{l}0.04 \\
0.06 \\
0.08 \\
0.1 \\
0.15 \\
0.18 \\
0.2\end{array}$ & $\begin{array}{l}0.2 \\
0.2 \\
0.2 \\
0.2 \\
0.2 \\
0.2 \\
0.2\end{array}$ & $\begin{array}{l}\text { C.H. } \\
\text { C.H. } \\
\text { C.H. } \\
\text { C.H. } \\
\text { C.H. } \\
\text { C.H. } \\
\text { S.I.H. }\end{array}$ & $\begin{array}{l}\text { C.H. } \\
\text { C.H. } \\
\text { C.H. } \\
\text { C.H. } \\
\text { C.H. } \\
\text { C.H. } \\
\text { C.H. }\end{array}$ & $\begin{array}{l}\text { C.H. } \\
\text { C.H. } \\
\text { C.H. } \\
\text { C.H. } \\
\text { C.H. } \\
\text { C.H. } \\
\text { S.I.H. }\end{array}$ & $\begin{array}{c}\text { C.H. } \\
\text { C.H. } \\
\text { C.H. } \\
\text { C.H. } \\
\text { S.I.H. } \\
\text { M.I.H. } \\
\text { I.H. }\end{array}$ & $\begin{array}{l}\text { C.H. } \\
\text { C.H. } \\
\text { C.H. } \\
\text { C.H. } \\
\text { S.I.H. } \\
\text { M.I.H. } \\
\text { I.H. }\end{array}$ & $\begin{array}{l}\text { C.H. } \\
\text { C.H. } \\
\text { C.H. } \\
\text { C.H. } \\
\text { C.H. } \\
\text { C.H. } \\
\text { S.I.H. }\end{array}$ & $\begin{array}{l}\text { C.H. } \\
\text { C.H. } \\
\text { C.H. } \\
\text { C.H. } \\
\text { C.H. } \\
\text { C.H. } \\
\text { C.H. }\end{array}$ & $\begin{array}{l}\text { C.H. } \\
\text { C.H. } \\
\text { C.H. } \\
\text { S.I.H. } \\
\text { S.I.H. } \\
\text { M.I.H. } \\
\text { M.I.H. }\end{array}$ \\
\hline
\end{tabular}

TABLE I 2.

Antigen of aceton insoluble lipoids; antigenic with syphilitic antibody in amounts of 0.1 c.c.; becomes anti-complementary in amounts of $0.45 \mathrm{c.c}$.

\begin{tabular}{|c|c|c|c|c|c|c|c|c|c|}
\hline $\begin{array}{l}\text { Serum } \\
\text { c.c. }\end{array}$ & $\begin{array}{l}\text { Amount of } \\
\text { Antigen c.c. }\end{array}$ & $\underset{\mathbf{I}}{\operatorname{Serum}}$ & $\underset{z}{\text { Serum }}$ & $\underset{3}{\text { Serum }}$ & $\begin{array}{l}\text { Serum } \\
4\end{array}$ & $\begin{array}{c}\text { Serum } \\
5\end{array}$ & $\underset{6}{\text { Serum }}$ & $\begin{array}{c}\text { Serum } \\
7\end{array}$ & $\underset{8}{\text { Serum }}$ \\
\hline $\begin{array}{l}0.04 \\
0.06 \\
0.08 \\
0.1 \\
0.15 \\
0.18 \\
0.2\end{array}$ & $\begin{array}{l}\text { O.I } \\
\text { O.I } \\
\text { O.I } \\
\text { O.I } \\
\text { o.I } \\
\text { O.I } \\
0 . I\end{array}$ & $\begin{array}{l}\text { C.H. } \\
\text { C.H. } \\
\text { C.H. } \\
\text { C.H. } \\
\text { C.H. } \\
\text { C.H. }\end{array}$ & $\begin{array}{l}\text { C.H. } \\
\text { C.H. } \\
\text { C.H. } \\
\text { C.H. } \\
\text { C.H. } \\
\text { C.H. } \\
\text { C.H. }\end{array}$ & $\begin{array}{l}\text { C.H.H. } \\
\text { C.H. } \\
\text { C.H. } \\
\text { CH. } \\
\text { C.H. } \\
\text { C.H. } \\
\text { C.H. }\end{array}$ & $\begin{array}{l}\text { C.H. } \\
\text { C.H. } \\
\text { C.H. } \\
\text { C.H. } \\
\text { C.H. } \\
\text { C.H. } \\
\text { S.I.H. }\end{array}$ & $\begin{array}{l}\text { C.H. } \\
\text { C.H. } \\
\text { C.H. } \\
\text { C.H. } \\
\text { S.I.H. } \\
\text { M.I.H. } \\
\text { I.H. }\end{array}$ & $\begin{array}{l}\text { C.H. } \\
\text { C.H. } \\
\text { C.H. } \\
\text { C.H. } \\
\text { C.H. } \\
\text { S.I.H.H. } \\
\text { M.I.H. }\end{array}$ & $\begin{array}{l}\text { C.H. } \\
\text { C.H. } \\
\text { C.H. } \\
\text { C.H. } \\
\text { C.H. } \\
\text { C.H. } \\
\text { C.H. }\end{array}$ & $\begin{array}{l}\text { C.H. } \\
\text { C.H. } \\
\text { C.H. } \\
\text { S.I.H. } \\
\text { M.I.H. } \\
\text { I.H. } \\
\text { I.H. }\end{array}$ \\
\hline
\end{tabular}

These results indicate that the diphtheria bacillus causes the production of a body capable of fixing complement only with its 
homologous antigen. The results with the two antigens of syphilitic liver and aceton insoluble lipoids were quite similar and with the exception of serum No. 8 showed uniform lack of power of fixing complement with the diphtheria antibody present in the immune sera. However, as already shown, the complement-fixing body of the diphtheria bacillus is the same for all types, including the Hofmann's bacillus, which organism is believed by some to be a separate entity.

In order to test still further the relation between diphtheria antigens and their immune sera, typhoid and streptococcus sera were tested with the eight different diphtheria antigens in order to ascertain whether the immune bodies present in these sera were capable of fixing complement with the diphtheria antigens:

TABLE $\mathrm{r}_{3}$.

Typhoid immune serum; agglutinates in dilution of $\mathrm{I}$ : 1,500 (macroscopical test); is only slightly antigenic with its own antigen. Tested with the eight diphtheria antigens as follows:

\begin{tabular}{|c|c|c|c|c|c|c|c|c|c|}
\hline $\begin{array}{c}\text { Serum } \\
\text { c.c. }\end{array}$ & $\begin{array}{l}\text { Antigen } \\
\text { c.c. }\end{array}$ & $\underset{I}{\text { Antigen }}$ & $\begin{array}{c}\text { Antigen } \\
2\end{array}$ & $\underset{3}{\text { Antigen }}$ & ${\underset{4}{4}}^{\text {Antigen }}$ & $\underset{5}{\text { Antigen }}$ & $\underset{6}{\text { Antigen }}$ & $\underset{7}{\text { Antigen }}$ & $\underset{8}{\text { Antigen }}$ \\
\hline $\begin{array}{l}0.04 \\
0.06 \\
0.08 \\
0.1 \\
0.15 \\
0.18 \\
0.2\end{array}$ & $\begin{array}{l}0.08 \\
0.08 \\
0.08 \\
0.08 \\
0.08 \\
0.08 \\
0.08\end{array}$ & $\begin{array}{l}\text { C.H. } \\
\text { C.H. } \\
\text { C.H. } \\
\text { C.H. } \\
\text { C.H. } \\
\text { C.H. } \\
\text { C.H. }\end{array}$ & $\begin{array}{l}\text { C.H. } \\
\text { C.H. } \\
\text { C.H. } \\
\text { C.H. } \\
\text { C.H. } \\
\text { C.H. } \\
\text { S.I.H. }\end{array}$ & $\begin{array}{l}\text { C.H. } \\
\text { C.H. } \\
\text { C.II. } \\
\text { C.H. } \\
\text { C.H. } \\
\text { C..H. } \\
\text { C.H. }\end{array}$ & $\begin{array}{l}\text { C.H. } \\
\text { C.H. } \\
\text { C.H. } \\
\text { C.H. } \\
\text { C.H. } \\
\text { C.H. } \\
\text { S.I.H. }\end{array}$ & $\begin{array}{l}\text { C.H. } \\
\text { C.H. } \\
\text { C.H. } \\
\text { C.H. } \\
\text { C.H. } \\
\text { C.H. } \\
\text { C.H. }\end{array}$ & $\begin{array}{c}\text { C.H. } \\
\text { C.H. } \\
\text { C.H. } \\
\text { C.H. } \\
\text { C.H. } \\
\text { S.l.H. } \\
\text { M.I.H. }\end{array}$ & $\begin{array}{l}\text { C.H. } \\
\text { C.H. } \\
\text { C.H. } \\
\text { C.H. } \\
\text { C..H. } \\
\text { C..H. } \\
\text { C.H. }\end{array}$ & $\begin{array}{l}\text { C.H. } \\
\text { C.H. } \\
\text { C.H. } \\
\text { C.H. } \\
\text { C.H. } \\
\text { C.H. } \\
\text { C.H. }\end{array}$ \\
\hline
\end{tabular}

TABLE I4.

Anti-streptococcus serum; produced by a large number of injections of 16 different strains of scarlet fever streptococci into a horse; the serum in amounts of 0.08 c.c. fixes complement with $0.09 \mathrm{c.c}$. of antigen of scarlet fever streptococci. Tested with the eight diphtheria antigens as follows:

\begin{tabular}{|c|c|c|c|c|c|c|c|c|c|}
\hline $\begin{array}{c}\text { Serum } \\
\text { c.c. }\end{array}$ & $\begin{array}{l}\text { Amount of } \\
\text { Antigen c.c. }\end{array}$ & $\underset{I}{\text { Antigen }}$ & $\underset{2}{\text { Antigen }}$ & $\underset{3}{\text { Antigen }}$ & $\underset{4}{\text { Antigen }}$ & $\begin{array}{c}\text { Antigen } \\
5\end{array}$ & $\underset{6}{\text { Antigen }}$ & $\begin{array}{c}\text { Antigen } \\
7\end{array}$ & $\underset{8}{\text { Antigen }}$ \\
\hline $\begin{array}{l}0.04 \\
0.06 \\
0.08 \\
0.1 \\
0.15 \\
0.18 \\
0.2\end{array}$ & $\begin{array}{l}0.08 \\
0.08 \\
0.08 \\
0.08 \\
0.08 \\
0.08 \\
0.08\end{array}$ & $\begin{array}{c}\text { C.H. } \\
\text { C.H. } \\
\text { C.H. } \\
\text { C.H. } \\
\text { S.I.H. } \\
\text { S.I.H. } \\
\text { M.I.H. }\end{array}$ & $\begin{array}{c}\text { C.H. } \\
\text { C.H. } \\
\text { C.H. } \\
\text { C.H. } \\
\text { S.I.H. } \\
\text { S.I.H, } \\
\text { M.I.H. }\end{array}$ & $\begin{array}{c}\text { C.H. } \\
\text { C.I. } \\
\text { C.H. } \\
\text { C.H. } \\
\text { S.I.H. } \\
\text { M.I.H. } \\
\text { I.H. }\end{array}$ & $\begin{array}{c}\text { C.H. } \\
\text { C.H. } \\
\text { C.H. } \\
\text { C.H. } \\
\text { S.1.H. } \\
\text { M.1.H. } \\
\text { M.I.H. }\end{array}$ & $\begin{array}{c}\text { C.H. } \\
\text { C.H. } \\
\text { C.H. } \\
\text { C.H. } \\
\text { S.I.H. } \\
\text { M.I.H. } \\
\text { I.H. }\end{array}$ & $\begin{array}{c}\text { C.H. } \\
\text { C.H. } \\
\text { C.H. } \\
\text { C.H. } \\
\text { S.I.H. } \\
\text { M.I.H. } \\
\text { M.I.H. }\end{array}$ & $\begin{array}{c}\text { C.H. } \\
\text { C.H. } \\
\text { C.H. } \\
\text { C.H. } \\
\text { S.I.H. } \\
\text { M.I.H. } \\
\text { I.H. }\end{array}$ & $\begin{array}{c}\text { C.H. } \\
\text { C.H. } \\
\text { C.H. } \\
\text { C.H. } \\
\text { S.I.H. } \\
\text { M.I.H. } \\
\text { I.H. }\end{array}$ \\
\hline
\end{tabular}

An examination of these results shows that as a rule complement is not fixed by typhoid and streptococcus immune bodies and diphtheria antigens and that therefore the relation between diphtheria antigens and immune body is more or less specific. 
RELATION BETWEEN DEGREE OF COMPLEMENT-FIXATION AND ANTITOXIN CONTENT OF DIPHTHERIA IMMUNE SERA.

The results of complement-fixation tests with sera secured from three different antitoxin laboratories and nine different antigens of diphtheria bacilli, including the same bacilli used in the production of these antitoxins, shows no relation between the occurrence or degree of complement-fixation and the antitoxic strength of the serum. These studies were then extended along similar lines, using other organisms and their immune sera. The results of this study are given in a separate communication. While the number of antitoxin units in the eight sera used in these experiments was not determined, yet the results with sera of known strengths demonstrated clearly the lack of any relation between complement-fixation and antitoxin content and indicated that in complement-fixation we are probably dealing with a body in the serum, in the nature of an amboceptor, not a true antibody in the sense of being prophylactic or curative.

HOFMANN'S BACILLUS (PSEUDO-DIPHTHERIA BACILLUS).

Most of the controversy in the bacteriology of diphtheria concerns this organism. A great amount of work has been done in an effort to establish its identity and its relation to the true diphtheria bacillus. By various ways, including passage through different animals, growth upon different culture media, acid production with different sugars, and straining reactions, attempts were made to change the morphological, biological, and pathogenic properties of this organism to make it resemble the true granular types of diphtheria bacilli. Conversely, attempts were made to convert true diphtheria bacilli into the pseudo-types. As a rule the sum total of the various work recorded indicates that Hofmann's bacillus possesses such characteristics as to permit its differentiation from the true type of diphtheria bacilli. The results of this complementfixation work indicate that it belongs essentially to the diphtheria group and is not a separate entity in the sense of being totally unrelated. It would appear that this bacillus has been changed in its chemical reaction and structure, as a result of being hard pressed, and has gradually developed a state of effective resistance 
or "fastness" to unfavorable surroundings. The new qualities acquired may thus be considered the result of "mutation" (Flexner) among the members of this group of organisms. As a result an organism has been evolved, "a mutant," capable of transmitting its new characteristics through an indefinite number of generations. The Hofmann bacillus is the best marked example of mutation among the diphtheria group, but as would be expected many other strains may be found with partially fixed characteristics, as, for instance, many of the strains of the $\mathrm{D}_{2}$ type which closely resemble Hofmann's bacillus with the exception of being able to produce acid with one or more of the sugars. To these the unfortunate and hopeless term "pseudo-diphtheria" has been applied. It would be better to call them non-virulent diphtheria bacilli.

CONCLUSIONS.

I. Complement-fixation reactions with homologous antigens and immune sera of different types of diphtheria bacilli from a variety of clinical conditions, including a true Hofmann's bacillus, tend to show that these organisms are all related.

2. The true Hofmann's bacillus is probably an example of "mutation" and is able to transmit its new qualities from generation to generation.

3. There is no relation between the occurrence or degree of complement-fixation and antitoxic strength of diphtheria immune sera. It seems that complement is fixed by a "body" separate from the true immune body representing the antitoxic content of sera. 